\title{
Solution analytique du problème de la réfraction de la houle
}

\section{Analytical resolution of the swell refraction problem}

par P.M. Petridis

Marine Technology Development, Grèce

This paper deals with the swell refraction in a less deep area, where the swell speed is affected by the deepness decrease. The analytical resolution proposed uses the Snell's law.

\section{I $\square$ LI DE BASE DE L'ANALYSE}

L'analyse utilise la loi de Snell. Si les lignes de niveau sont des droites parallèles, la validité de la loi pour toute profondeur peut être prouvée théoriquement sur la base de l'équation différentielle de phase (« eiconal equation»). La formulation mathématique de la loi est la suivante :

$\sin a / C=\sin \alpha_{0} / C_{0}=c($ constante $)=\left(2 n \sin \alpha_{0}\right) /(g T \tanh \pi)$

où

$C, C_{0}, \alpha, \alpha_{0}$ sont respectivement les célérités de phase de la houle à la profondeur $d_{0}, d$, et les angles entre la direction de la houle et la normale sur la ligne de niveau de profondeur $d_{0}, d, T$ est la période de la houle (constante) et $g$ est l'accélération de la gravité.

\section{EÉQUATION DIFFÉRENTIELLE DES COURBES ORTHOGONALES}

Sur la base de la loi de Snell et pour une géométrie simple l'équation différentielle des courbes orthogonales prend la forme :

$$
x=C \cdot \int \frac{C(y) d y}{\sqrt{1-c^{2} C^{2}(y)}}+c_{0}
$$

La constante $c_{0}$ de l'orthogonale dépend du système de référence.

Considérant la fonction du fond $z=f(y)$ et substituant la célérité de phase d'après la formule d'Airy, la forme para- métrique suivante de l'équation des orthogonales (avec la célérité de phase $C$ comme paramètre) dérive :

$X=C . \int \frac{C Y_{Z}^{*}(Z)}{\sqrt{1-c^{2} C^{2}}} \cdot\left[\frac{T}{4 \pi} \ln \frac{g T+2 \pi C}{g T-2 \pi C}+\frac{g T^{2} C}{g T^{2}-4 \pi^{2} C^{2}}\right] d C+c_{0}$

Après l'intégration, il résulte :

$$
\begin{aligned}
& x=F\left(C, c_{0}\right)=F(C, 0)+c_{0} \\
& y=y\left(f_{0}(C)\right)=\phi(C)
\end{aligned}
$$

III $\square$ SOLUTION ANALYTIQUE POUR UN FOND D'INCLINAISON CONSTANTE ET UNE PROFONDEUR INTERMÉDIAIRE ET FAIBLE

Après une longue série de manipulations, l'intégration de (3) conduit aux relations finales suivantes qui constituent la solution paramétrique de l'équation des orthogonales: Cas $a: \alpha_{0}<\sin ^{-1}(\tanh \pi)$

$$
X=\frac{\cot \varphi}{2 a g C^{2}}\left\{\operatorname { l n } \left\{\left(\frac{1-a c C}{1+a c C}\right)^{\sqrt{1-c^{2} c^{2}}} .\right.\right.
$$

$$
\left(\frac{\sqrt{1-c^{2} C^{2}}+\sqrt{a^{2}-1} \cdot c \cdot C}{\sqrt{1-c^{2} C^{2}}-\sqrt{a^{2}-1} \cdot c \cdot C}\right)^{1 / a \sqrt{a^{2}-1}} .
$$




$$
\begin{aligned}
& {\left.\left.\left[\frac{\left(1-\sqrt{1-c^{2} C^{2}}\right)\left(1-c C \sqrt{a^{2}-1}\right)-c^{2} C^{2}}{\left(1-\sqrt{1-c^{2} C^{2}}\right)\left(1+c c \sqrt{a^{2}-1}\right)-c^{2} C^{2}}\right]^{\sqrt{a^{2}-1}}\right]\right\}+C_{0} . } \\
= & \frac{\cot \varphi \cdot a g}{2 \omega^{2}}\left\{\operatorname { l n } \left\{\left(\frac{1-a \sin \alpha}{1+a \sin \alpha}\right)^{\cos \alpha} \cdot\left(\frac{\cos \alpha+\sqrt{a^{2}-1} \sin \alpha}{\cos \alpha-\sqrt{a^{2}-1} \sin \alpha}\right)^{1 / a \sqrt{a^{2}-1}} .\right.\right. \\
= & {\left.\left.\left[\frac{(1-\cos \alpha)\left(1-\sin \alpha \sqrt{a^{2}-1}-\sin ^{2} \alpha\right)}{(1-\cos \alpha)\left(1+\sin \alpha \sqrt{a^{2}-1}-\sin ^{2} \alpha\right)}\right]^{\sqrt{a^{2}-1} / a}\right\}\right\}+c_{0} } \\
= & F_{1}\left(\mathrm{C}, \mathrm{c}_{0}\right)=F_{1}^{*}\left(\alpha, c_{0}\right)
\end{aligned}
$$

Cas $b: \alpha_{n}<\sin ^{-1}(\tanh \pi)$

$$
\begin{aligned}
& x=\frac{\cot \varphi}{2 a g C^{2}}\left\{\ln \left(\frac{1-a c C}{1+a c C}\right)^{\sqrt{1-c^{2} C^{2}}}\right. \\
& \quad+2\left[\frac{1}{a \sqrt{1-a^{2}}} \tan ^{-1} \frac{\sqrt{1-a^{2} c C}}{\sqrt{1-c^{2} C^{2}}}\right. \\
& \left.\left.-\frac{\sqrt{1-a^{2}}}{a} \tan ^{-1}\left(\frac{c C \sqrt{1-a^{2}}\left(1-\sqrt{1-c^{2} C^{2}}\right)}{c^{2} C^{2}-\left(1-\sqrt{1-c^{2} C^{2}}\right)}\right)\right]\right\}+c_{0} \\
& =\frac{\cot \varphi \cdot a g}{2 \omega^{2}}\left\{\ln \left(\frac{1-a \sin \alpha}{1+a \sin \alpha}\right)^{\cos \alpha}\right.
\end{aligned}
$$$$
+2 \frac{1}{a \sqrt{1-a^{2}}} \tan ^{-1}\left(\sqrt{1-a^{2}}+\tan \alpha\right)-
$$$$
\left.-\frac{\sqrt{1-a^{2}}}{a} \cdot \tan ^{-1}\left(\frac{\sin \alpha \sqrt{1-a^{2}}(1-\cos \alpha)}{\sin ^{2} \alpha-(1-\cos \alpha)}\right)\right\}+C_{0}
$$$$
=F_{2}\left(\mathrm{C}, \mathrm{c}_{0}\right)=F_{2}^{*}\left(C, c_{0}\right)
$$

Cas $c: \alpha_{0}<\sin ^{-1}(\tanh \pi)$

$$
X=\frac{\cot \varphi}{2 g c^{2}} \ln \left(\frac{1-c C}{1+c C}\right)^{\sqrt{1-c^{2} C^{2}}}+\frac{2 c C}{\sqrt{1-c^{2} C^{2}}}+c_{0}
$$

$$
\begin{aligned}
& =\frac{\cot \varphi a g}{2 \omega^{2}} \cdot \ln \left(\frac{1-\sin \alpha}{1+\sin \alpha}\right)^{\cos \alpha}+2 \tan \alpha+c_{0} \\
& =F_{3}(C)=F_{3}^{*}(\alpha)
\end{aligned}
$$

pour tous les cas

$$
\begin{aligned}
y & =\cot \varphi \cdot \frac{C T}{4 n} \cdot \ln \frac{g T+2 \pi C}{g T-2 \pi C}=\frac{\cot \varphi \cdot a \cdot g}{2 \omega^{2}} \cdot \sin \alpha \ln \left(\frac{1+a \sin \alpha}{1-a \sin \alpha}\right) \\
& =\phi(C)=\phi^{*}(\alpha)
\end{aligned}
$$

où

$$
a=\tanh \pi / \sin \alpha_{0}
$$

$\varphi$ est l'angle d'inclinaison du fond.

\section{IV — PROFONDEUR VARIABLE}

En utilisant les équations précédemment dérivées et les conditions aux limites, pour chaque intervalle (i), la réfraction a lieu conformément aux équations :

$$
\begin{aligned}
& x=F_{k}\left(C, \varphi_{i}, 0\right)-F_{k}\left(C_{i}, \varphi_{i}, 0\right)+X_{0 i} \\
& y=\phi_{k}\left(C, \varphi_{i}\right)+A_{i}
\end{aligned}
$$

avec :

$$
C_{i-1}<C<C_{i}
$$

où :

$$
\begin{aligned}
& A_{i}=y_{i-1}-z_{i-1} \cot \varphi_{i}=\sum_{j=1}^{i-1} d_{j} \cot \varphi_{j}-\cot \varphi_{i}\left(\sum_{j=1}^{i-1} d_{j}\right) \\
& X_{0 i}=X_{i+1}-F_{k}\left(C_{i+1}, \varphi_{i+1}, 0\right)+F_{k}\left(C_{i}, \varphi_{i+1}, 0\right)
\end{aligned}
$$

où $x_{n}$ est connu.

Les valeurs de l'indice $k$ correspondent aux cas $(a)$, (b), (c) respectivement.

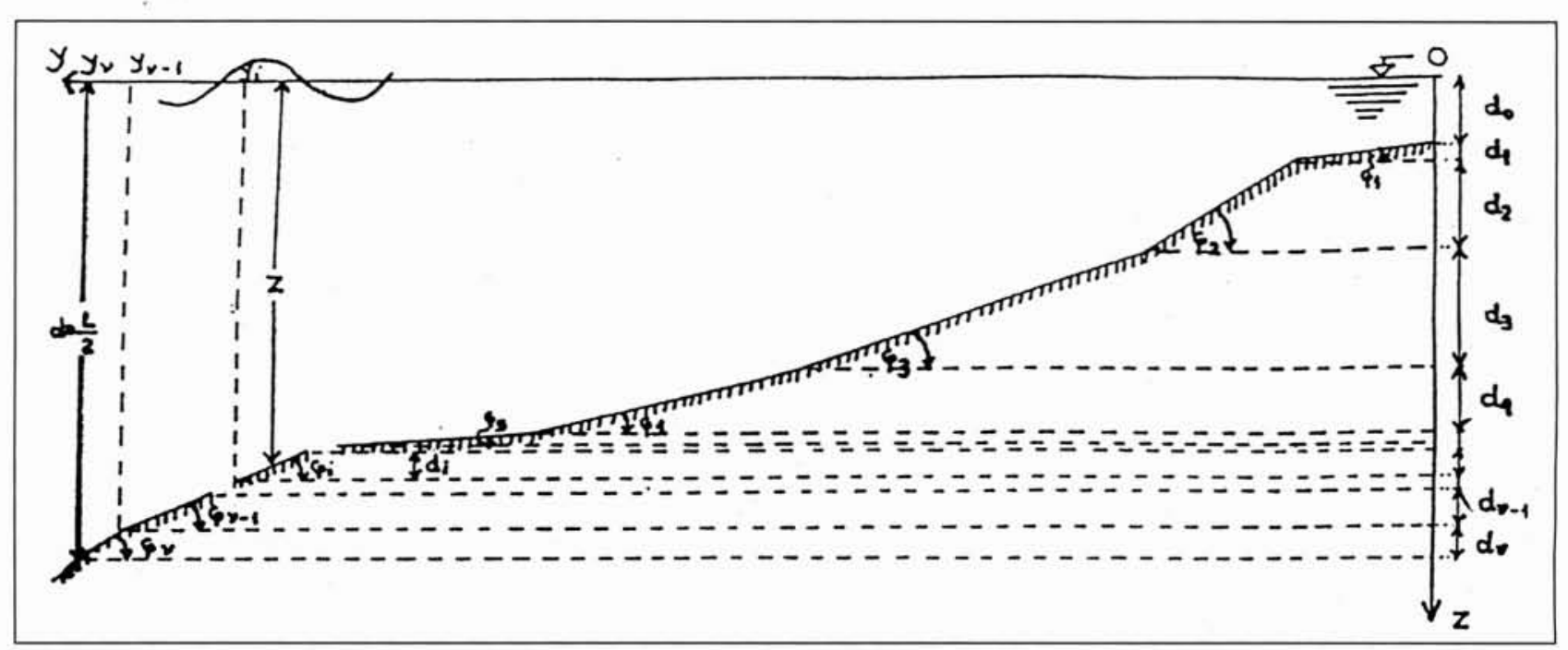

1. Section du fond avec des variations linéaires de la profondeur par segments. 


\section{Mécanique des fluides}

2. Comparaison des résultats des solutions analytique, numérique et graphique.

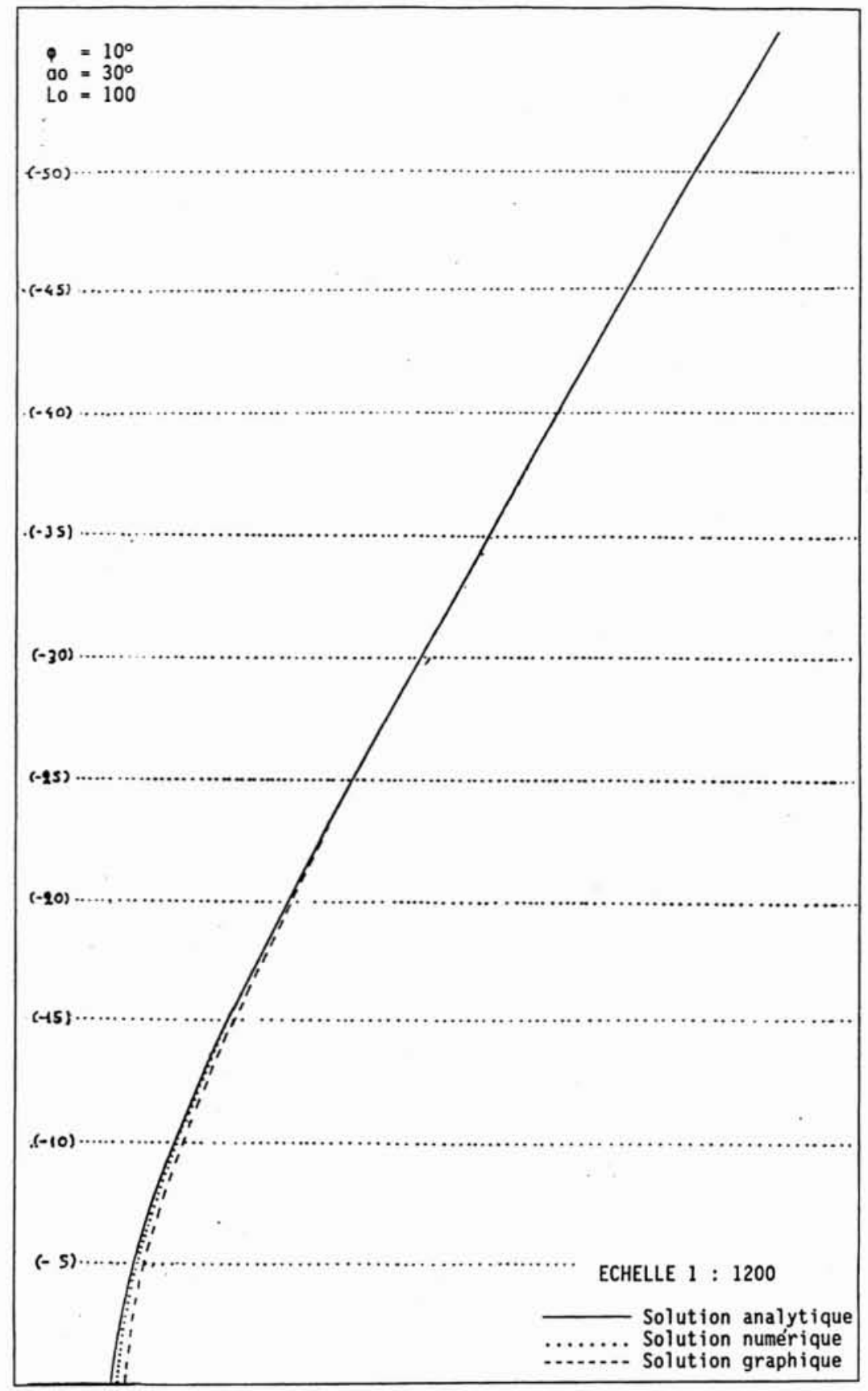

\section{V — PRÉSENTATION DES RÉSULTATS}

Dans le cas d'une variation linéaire de la profondeur, les résultats de la solution analytique du problème de la propagation de la houle sont présentés sur la figure 2 comparés avec les résultats d'une solution numérique (basée sur le schéma numérique de Griswold-Lepetit) et de la solution graphique.

Les données d'application sont les suivantes :

Angle d'inclinaison du fond :

Angle d'incidence de la houle à grande profondeur :

Longueur d'onde à grande profondeur :

$$
\begin{aligned}
& \varphi=10^{\circ} \\
& \alpha_{0}=30^{\circ} \\
& L_{0}=100 \mathrm{~m}
\end{aligned}
$$

Sur la figure 2, on constate une concordance très satisfaisante entre les solutions analytique et numérique. 